\title{
Kinetic and Structural Properties of a Robust Bacterial L-Amino Acid Oxidase
}

\author{
Simone Savino ${ }^{1,2} \mathbb{D}$, J. Daniël-Moráh Meijer ${ }^{2}$, Henriëtte J. Rozeboom ${ }^{2}$, Hugo L. van Beek ${ }^{1}$ \\ and Marco W. Fraaije 2 ,*(D) \\ 1 GECCO Biotech, Nijenborgh 4, 9747 AG Groningen, The Netherlands; s.savino@rug.nl (S.S.); \\ h.l.van.beek@gecco-biotech.com (H.L.v.B.) \\ 2 Molecular Enzymology Group, Groningen Biomolecular Sciences and Biotechnology Institute, University of \\ Groningen, Nijenborgh 4, 9747 AG Groningen, The Netherlands; j.d.m.meijer@rug.nl (J.D.-M.M.); \\ h.j.rozeboom@rug.nl (H.J.R.) \\ * Correspondence: m.w.fraaije@rug.nl
}

Citation: Savino, S.; Meijer, J.D.-M.; Rozeboom, H.J.; van Beek, H.L.; Fraaije, M.W. Kinetic and Structural Properties of a Robust Bacterial L-Amino Acid Oxidase. Catalysts 2021, 11, 1309. https://doi.org/ $10.3390 /$ catal11111309

Academic Editors:

Evangelos Topakas, David D. Boehr and Roland Wohlgemuth

Received: 12 October 2021

Accepted: 27 October 2021

Published: 28 October 2021

Publisher's Note: MDPI stays neutral with regard to jurisdictional claims in published maps and institutional affiliations.

Copyright: (c) 2021 by the authors. Licensee MDPI, Basel, Switzerland. This article is an open access article distributed under the terms and conditions of the Creative Commons Attribution (CC BY) license (https:/ / creativecommons.org/licenses/by/ $4.0 /)$.

\begin{abstract}
L-Amino acid oxidase (LAAO) is a flavin adenine dinucleotide (FAD)-dependent enzyme active on most proteinogenic L-amino acids, catalysing their conversion to $\alpha$-keto acids by oxidative deamination of the substrate. For this oxidation reaction, molecular oxygen is used as the electron acceptor, generating hydrogen peroxide. LAAO can be used to detect L-amino acids, for the production of hydrogen peroxide as an oxidative agent or antimicrobial agent, and for the production of enantiopure amino acids from racemates. In this work, we characterised a previously reported LAAO from the bacterium Pseudoalteromonas luteoviolacea. The substrate scope and kinetic properties of the enzyme were determined, and the thermostability was evaluated. Additionally, we elucidated the crystal structure of this bacterial LAAO, enabling us to test the role of active site residues concerning their function in catalysis. The obtained insights and ease of expression of this thermostable LAAO provides a solid basis for the development of engineered LAAO variants tuned for biosensing and/or biocatalysis.
\end{abstract}

Keywords: flavin-dependent oxidase; L-amino acids; deracemisation; biocatalysis; crystal structure

\section{Introduction}

LAAOs are flavin-dependent enzymes present in many organisms [1]. Until recently, representatives were only known and isolated from eukaryotes. LAAOs have been isolated from various snakes and are often a major component in venom [2] and given these venomous secretions their typical yellow colour [3]. Studies have revealed that these proteins consist of three structural elements: a substrate-binding domain, an FAD domain, and a helical domain [4]. LAAOs catalyse the oxidative deamination of L-amino acids to $\alpha$-keto acids, and substrate ranges have been determined for different LAAOs [5].

LAAOs harbour a tightly bound FAD cofactor which assists in catalysis. The mechanism can be divided in two discrete catalytic steps (Figure 1). Initially, the amino acid substrate is oxidised to the imino acid, which spontaneously reacts with water to form the $\alpha$-keto acid and ammonium. This results in the formation of the flavin adenine dinucleotide (FAD) cofactor in the reduced state $\left(\mathrm{FADH}_{2}\right)$. Regeneration of the reduced FAD by molecular oxygen leads to the production of hydrogen peroxide. It is the formation of this reactive oxygen species that has been the focus of studies concerning the role of LAAOs as inducers of oxidative stress [6]. The oxidative damage induced by LAAO activity from snake venom leads to cytotoxicity, antibacterial activity, and the disruption of platelet aggregation [7-9]. Recent biochemical studies have revealed that LAAOs are not only present in the venom of snakes but are also produced in other eukaryotes and even bacteria [1]. 


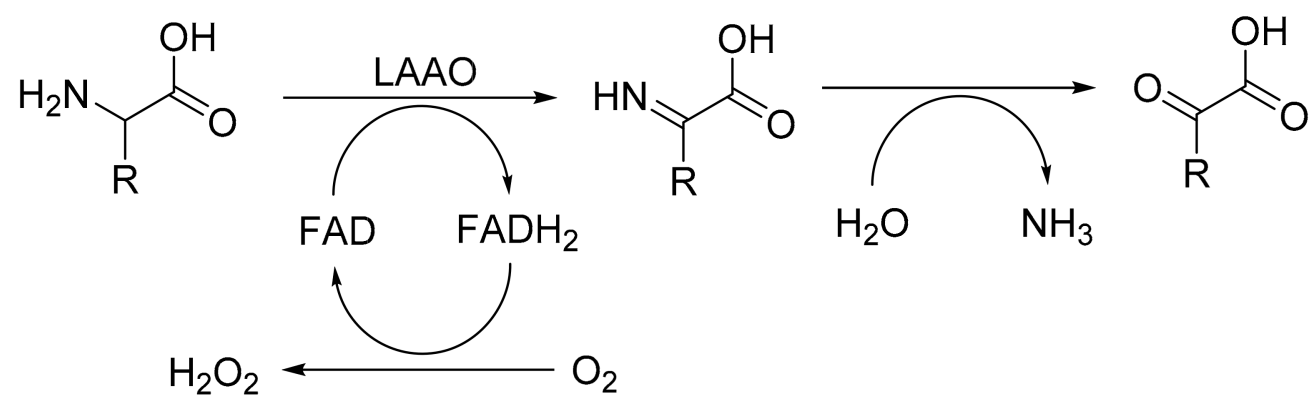

Figure 1. The catalytic mechanism of LAAO: oxidation of amino acids into imino acids and the subsequent reoxidation of the FAD cofactor by reduction of dioxygen into hydrogen peroxide. The hydration of the initial product, imino acid, is a spontaneous reaction.

LAAOs are regarded as valuable biotechnological tools. Applications include their use as biosensors to detect amino acids [10], as antibacterial agents, and as biocatalysts [11]. As a biocatalytic tool, LAAO can be applied to deracemise amino acids, generating enantiopure $\mathrm{D}$-amino acids. This approach takes advantage of an almost exclusive activity of LAAO towards L-amino acids, leaving D-amino acids untouched. Through in situ reduction of the imino acid intermediate produced in the first step of the reaction using a chemical reductant, enantiopure D-amino acids can be produced [12]. LAAO can also be considered for alternative biocatalytic routes. A more recent example of the use of an LAAO in biocatalysis was described by Busch et al. [13], where it was used for the in situ generation of $\alpha$-ketoglutarate using L-glutamate.

LAAO from the marine bacterium Pseudoalteromonas luteoviolacea (Pl-LAAO, 653 amino acids, $74 \mathrm{kDa}$ ) was shown to display antibacterial activity [14]. The reported broad substrate range of this enzyme makes it an attractive biocatalyst. We decided to investigate its biochemical features in more detail, which included the elucidation of its molecular structure. The obtained structure was analysed and compared with a recently reported structure of an ancestrally reconstructed LAAO, AncLAAO $[15,16]$. The determined structure of $P l$-LAAO was also used to design, create, and analyse several $P l$-LAAO mutants that revealed the role of specific active site residues.

\section{Results}

\subsection{Expression and Purification}

The final yield of $\mathrm{Pl}$-LAAO was approximately $200 \mathrm{mg} / \mathrm{L}$ of Escherichia coli culture in terrific broth (TB). This shows that this recombinant bacterial LAAO is well expressed as His-tagged protein, in contrast to previous attempts to express bacterial LAAOs [17]. It also outperforms the expression levels of recently reported ancestral reconstructed LAAOs (40-80 mg/L) [16]. Pl-LAAO was purified using immobilised metal affinity chromatography (IMAC) as shown by SDS-PAGE (Supplementary Figure S1). The UV-Vis absorbance spectrum of purified $\mathrm{Pl}$-LAAO shows absorbance features that are typical for a flavincontaining protein, in line with its yellow appearance (Supplementary Figure S2). The extinction coefficient of the FAD-bound enzyme was determined to be $11.4 \mathrm{mM}^{-1} \mathrm{~cm}^{-1}$ at $455 \mathrm{~nm}$ and used to quantify $P l$-LAAO. For crystallisation experiments, $P l$-LAAO was further purified using size exclusion chromatography, yielding a single peak corresponding to a monodispersed sample of dimeric Pl-LAAO (Supplementary Figure S3). This fits the observation of Andreo-Vidal et al. [14] that $P l$-LAAO is only active as an oligomeric protein and is in accordance with the oligomeric states previously reported for bacterial LAAOs [17].

\subsection{Thermostability}

To establish optimal conditions for studying and applying $\mathrm{Pl}$-LAAO, the thermostability of $P l$-LAAO was tested in several buffers and at different $\mathrm{pH}$ values. Interestingly, the apparent melting temperatures at $\mathrm{pH} 5-6.5(50 \mathrm{mM}$ Tris- $\mathrm{HCl})$ were found to be rela- 
tively high: $82-84{ }^{\circ} \mathrm{C}$ (Supplementary Figure S4a). At higher $\mathrm{pH}$, the stability decreased $\left(\mathrm{T}_{\mathrm{m}}=60^{\circ} \mathrm{C}\right.$ in $50 \mathrm{mM}$ Tris- $\left.\mathrm{HCl}, \mathrm{pH} 7.5\right)$. The addition of salt fully rescued the stability of Pl-LAAO at $\mathrm{pH} 7.5\left(\mathrm{~T}_{\mathrm{m}}=85^{\circ} \mathrm{C}\right.$ in $500 \mathrm{mM} \mathrm{NaCl}, 50 \mathrm{mM}$ Tris-HCl, $\mathrm{pH}$ 7.5) (Supplementary Figure $\mathrm{S} 4 \mathrm{~b})$.

To verify that the enzyme was not only retaining its structure at high temperatures but also remained functional, its residual activity was measured after incubation at various temperatures for $10 \mathrm{~min}$. The remaining activity of samples in $500 \mathrm{mM} \mathrm{NaCl}, 50 \mathrm{mM}$ Tris- $\mathrm{HCl}, \mathrm{pH} 7.5$ was measured at elevated temperatures (Supplementary Figure S4c). Incubation at 70 and $80^{\circ} \mathrm{C}$ only led to partial inactivation ( $>60 \%$ remaining activity), but at $90{ }^{\circ} \mathrm{C}$, the residual activity dropped to about $10 \%$. Residual activity was also monitored at $37^{\circ} \mathrm{C}$ for an extended time period (Supplementary Figure S4d). When using $500 \mathrm{mM}$ $\mathrm{NaCl}, 50 \mathrm{mM}$ Tris- $\mathrm{HCl}, \mathrm{pH} 7.5$, this resulted in slow inactivation, with $50 \%$ remaining activity after $56 \mathrm{~h}$. Interestingly, these thermostability data reveal that this extant LAAO is significantly more stable when compared with ancestrally reconstructed LAAOs $[15,16]$. The most stable ancestral LAAO lost most of its activity in $10 \mathrm{~min}$ at $70{ }^{\circ} \mathrm{C}(<20 \%$ remaining activity) and in $10 \mathrm{~h}$ at $30^{\circ} \mathrm{C}$ (about $30 \%$ remaining activity).

\subsection{Kinetic Properties}

Next, the kinetic parameters of $P l$-LAAO were determined for all 20 naturally occurring amino acids (Table 1, Supplementary Figure S5). No significant activity was detected in the case of proline and cysteine. Gratifyingly, oxidase activity was observed for all other L-amino acids. The highest rates were found for L-leucine and L-glutamine. This is in full agreement with the initial characterisation of this bacterial LAAO that only reported activities on L-amino acids at concentrations of $2 \mathrm{mM}$ [14]. We have found that $\mathrm{Pl}$-LAAO is most efficient with non-charged bulky L-amino acids (L-leucine, L-methionine, L-phenylalanine, L-glutamine, L-tryptophan) with $k_{\text {cat }}$ values of $36-136 \mathrm{~s}^{-1}$ and $\mathrm{K}_{\mathrm{m}}$ values ranging from 0.34 to $2.4 \mathrm{mM}$. There are also other L-amino acids with high $k_{\text {cat }}$ values (L-glutamate, L-isoleucine, L-arginine, L-valine, L-histidine, L-alanine, L-lysine) but they suffer from relatively high $\mathrm{K}_{\mathrm{m}}$ values. Other L-amino acids (mainly small and polar amino acids) displayed poor catalytic efficiencies. The substrate specificity resembles those of the recently reported ancestral LAAOs $[15,16]$ which were described to be most efficient with L-glutamine, L-phenylalanine, and L-methionine. The kinetic parameters of the most stable AncLAAO (AncLAAO-N4) for L-methionine $\left(k_{\text {cat }}=34.2 \mathrm{~s}^{-1}, \mathrm{~K}_{\mathrm{m}}=0.76 \mathrm{mM}\right.$ [16] were found to be similar to $P l$-LAAO $\left(k_{\text {cat }}=63 \mathrm{~s}^{-1}, \mathrm{~K}_{\mathrm{m}}=0.42 \mathrm{mM}\right.$, Table 1).

\subsection{Structure Elucidation}

The crystal structure of $P l$-LAAO was solved at $2.8 \AA$ resolution. The crystallographic data and the model refinement values are reported in Supplementary Table S1. The content of the asymmetric unit is the physiological dimer of $\mathrm{Pl}$-LAAO, with both molecules binding one FAD (Figure 2a). The structure of the Pl-LAAO monomer reveals an overall conserved folding with respect to the recently reported crystal structure of the ancestral LAAO, AncLAAO-N5 [16], with an rmsd value of $0.77 \AA$ when the monomers of each protein are superimposed. This is in line with the $71 \%$ sequence identity between both protein sequences. Two main differences are (i) the identity of surface residues, specifically some secondary structure elements exposed to the solvent and involved in the dimerisation interface (Supplementary Figure S6), and (ii) the number of intra-chain salt bridges (20 in $\mathrm{Pl}$-LAAO and 17 in AncLAAO). These differences in structural features may explain the relatively high stability of $\mathrm{Pl}$-LAAO. 
Table 1. Steady state kinetic parameters for amino acid substrates, ordered from highest to lowest $k_{\text {cat }} / \mathrm{K}_{\mathrm{m}}$ values. For L-glutamate, L-aspartate, and L-tyrosine, the individual kinetic parameters, $k_{\text {cat }}$ and $K_{m}$, could be determined because of the relatively high $K_{m}$ values in combination with relatively low solubility of some of these substrates.

\begin{tabular}{ccccc}
\hline Substrate & $\begin{array}{c}\boldsymbol{k}_{\text {cat }} \\
\left(\mathbf{s}^{-\mathbf{1}}\right)\end{array}$ & $\begin{array}{c}\mathbf{K}_{\mathbf{m}} \\
(\mathbf{m M} \mathbf{M})\end{array}$ & $\begin{array}{c}\boldsymbol{k}_{\mathbf{c a t}} / \mathbf{K}_{\mathbf{m}} \\
\left(\mathbf{s}^{-\mathbf{1}} \mathbf{m} \mathbf{M}^{-\mathbf{1}}\right)\end{array}$ & $\begin{array}{c}\mathbf{V}_{\mathbf{m a x}} \\
\mathbf{( U / m} \mathbf{m})\end{array}$ \\
\hline L-leucine & 72 & 0.40 & 180 & 58 \\
L-methionine & 63 & 0.42 & 150 & 51 \\
L-phenylalanine & 52 & 0.34 & 150 & 42 \\
L-glutamine & 136 & 2.4 & 56 & 110 \\
L-tryptophan & 36 & 1.5 & 25 & 29 \\
L-glutamate & $>30$ & $>6.9$ & 4.4 & 24 \\
L-isoleucine & 25 & 11 & 2.3 & 35 \\
L-arginine & 43 & 25 & 1.7 & 7.5 \\
L-valine & 9.4 & 9.5 & 0.99 & 4.3 \\
L-histidine & 5.3 & 11 & 0.48 & 11.3 \\
L-alanine & 14 & 48 & 0.30 & 5.8 \\
L-lysine & 7.2 & 57 & 0.13 & 0.17 \\
L-serine & 0.21 & 14 & 0.015 & 0.60 \\
L-tyrosine & $>0.75$ & $>10$ & 0.075 & 0.37 \\
L-asparagine & 0.46 & 8.7 & 0.053 & 0.018 \\
L-aspartate & $>0.023$ & $>3.8$ & 0.0061 & 0.040 \\
L-threonine & 0.05 & 14 & 0.0035 & 0.0081 \\
L-glycine & 0.01 & 30 & 0.0003 & \\
\hline
\end{tabular}
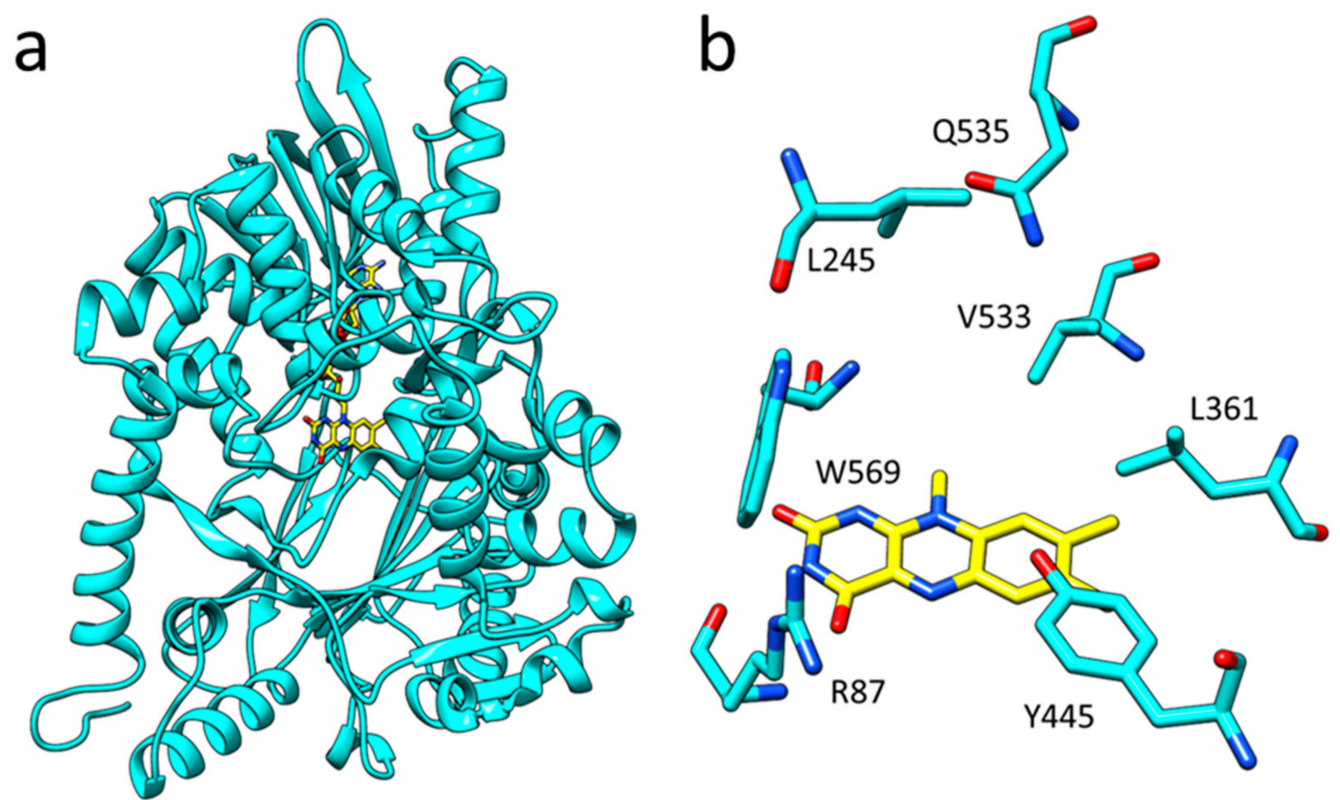

Figure 2. (a) The overall structure of $P l$-LAAO monomer in cyan ribbons and the FAD cofactor highlighted in yellow sticks. (b) Close-up of the active site, with the isoalloxazine moiety of the flavin cofactor in yellow and active site residues in cyan.

In one of the monomers, a glycerol molecule occupies the pocket that is next to the redox active part of the bound FAD, identifying the active site at the $R e$-face of the flavin cofactor (Supplementary Figure S7). Comparison with the L-phenylalanine-complexed structure of AncLAAO confirms the location of the substrate binding pocket. There is a tunnel leading from the solvent exposed surface to the substrate binding pocket (Supplementary Figure S8). A number of hydrophobic residues line the substrate binding pocket of Pl-LAAO (L245, L361, V533, W569) and account for the preference of Pl-LAAO for bulky 
hydrophobic amino acids (Figure 2b). There is one polar residue (Q535) located in the back of the binding pocket which explains the relatively good activity and affinity of $P l$-LAAO for L-glutamine. The residues R87 and Y445, positioned close to the isoalloxazine moiety of the flavin cofactor, bind the carboxyl group of amino acid substrates. Their essential role in catalysis was confirmed by generating and testing R87M and Y445F Pl-LAAO mutants, which displayed drastically reduced activity on L-phenylalanine ( $<2 \%$ activity).

\section{Discussion}

We have found that $\mathrm{Pl}$-LAAO can be overexpressed in E. coli, yielding large amounts of FAD-containing, soluble, active enzyme. Surprisingly, such high levels of expression do not seem to affect the viability of the expression host, even though it is active on abundantly present L-amino acids. It was discovered that $\mathrm{Pl}$-LAAO is quite thermotolerant and shows a higher tolerance to temperature when compared with other microbial and ancestral reconstructed LAAOs [16]. The relatively high temperature tolerance and preference for high salt concentrations may reflect the conditions at which the oxidase is active in real life. It has been found that it is secreted by the original bacterial host, contributing to the antimicrobial activity of Pseudoalteromonas luteoviolacea [14]. Substrate profiling of Pl-LAAO revealed that it is active on most L-amino acids. Yet, it shows the highest activity and affinity towards bulky hydrophobic L-amino acids and L-glutamine. No activity or very poor activity is observed with small amino acids.

To understand the structural basis for the stability and the substrate preferences of $\mathrm{Pl}$-LAAO we set out to determine its crystal structure. The obtained structure sheds light on the relatively high stability of this enzyme with respect to close homologs, the recently reported ancestral LAAOs [16]. While the core of the protein is structurally highly similar to the ancestral LAAOs, the surface residues are not conserved, especially in some specific regions (Supplementary Figure S6). The residues involved in the dimerisation of LAAO differ by 26 substitutions between $P l$-LAAO and AncLAAO. The latter enzyme accumulates asparagine and lysine residues in hotspots (N520-N522-N524 and K341-K345-K346) which may prevent complementarity of charges between the two monomers, lowering the stability of the dimeric protein. Moreover, the difference in the number of intra-chain salt bridges might contribute to the difference in thermostability.

By inspecting the $P l$-LAAO structure and, specifically, the substrate binding site, being rather large and hydrophobic, it becomes clear why a specific set of L-amino acids is preferred, similar to the ancestral LAAO. Comparing $\mathrm{Pl}$-LAAO with the structures of other flavin-dependent amino acid oxidases provides some clues on how evolution has generated several solutions for oxidising amino acids with the help of a flavin cofactor. Snake venom LAAOs, for example LAAO from the Malayan pit viper, also have well-described substrate scopes, typically with low $K_{m}$ values for many amino acids $[2,18]$ and its crystal structure has been reported [19]. Both Pl-LAAO (or AncLAAO) and venom LAAO use the same configuration of residues for binding and positioning of their substrates (Figure $3 a, b$ ). In both types of LAAOs, an arginine and a tyrosine residue interact with the carboxyl group of the amino acid substrate. This array of $\mathrm{H}$-bonds together with the side chain of a tryptophan, which defines one boundary of the active site, orient the L-amino acids for hydride transfer to the N5 atom of the FAD cofactor. The other part of the substrate binding pocket, interacting with the side chain of the amino acid substrate, shows many differences, explaining the different substrate preferences (Figure 3a,b). Intriguingly, D-amino acid oxidase (DAAO) from yeast, acting on mirror-image substrates and having quite a different protein structure, also contains a tyrosine and arginine couple that binds the carboxylate of the amino acid substrate (Figure $3 \mathrm{c}$ ). For DAAO, the binding pocket accommodating the substrate amino acid side chain is orthogonal compared to LAAO, allowing binding of the stereoisomeric substrates, D-amino acids [20]. The structural dissimilarities indicate that LAAOs and DAAOs independently evolved and converged to perform the same reaction, oxidation of amino acids, using a similar active site arrangement. The structural insights into the basis of substrate binding in $\mathrm{Pl}$-LAAO and other amino acid oxidases is valuable 
to understand their catalytic mechanism and can inform future enzyme engineering efforts towards other substrates.
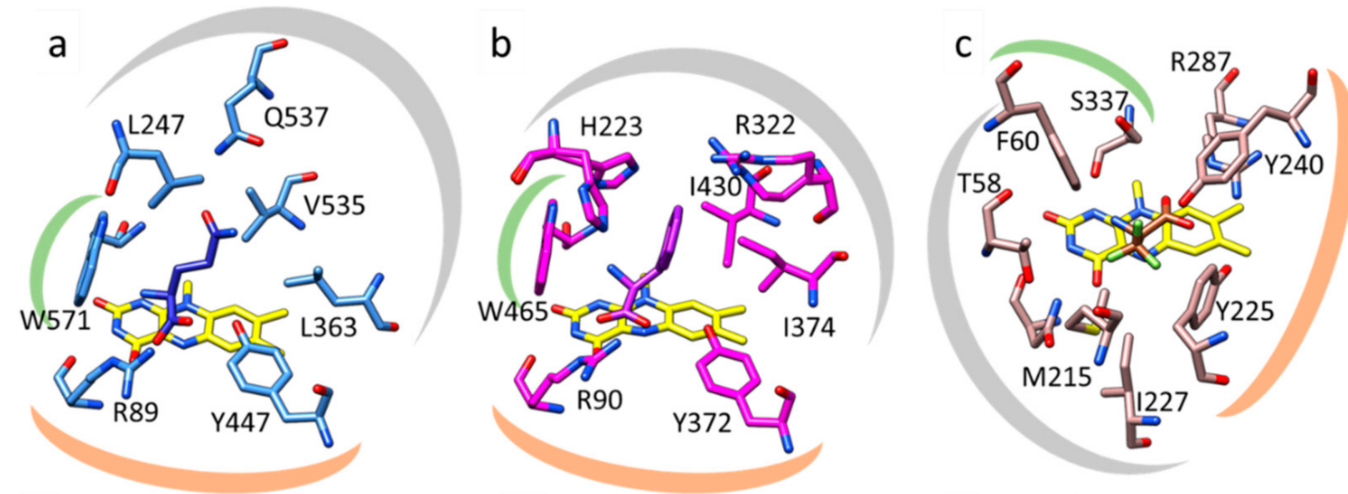

Figure 3. Structural comparison of active sites of amino acid oxidases. Active sites of (a) ancestral LAAO (7C4L), (b) LAAO from the Malayan pit viper (2IID), and (c) yeast DAAO (1C0L). In (a), residues R89 and Y447, highlighted by the orange mark, interact with the carboxyl group of the bound L-glutamine substrate (dark blue). In (b), the same role is exerted by R90 and Y372 for binding L-phenylalanine (purple). In (c), R287 and Y240 interact with the carboxyl moiety of the bound D-trifluoroalanine (dark brown). The green marks highlight the W571 and W465 in (a,b) respectively, which form part of the substrate binding pocket, close to the amine moiety of the bound substrate. In DAAO (c), a phenylalanine (F60) serves a similar role. The grey marks indicate the residues involved in binding of the substrate side chain in the three different amino acid oxidases.

\section{Materials and Methods}

\subsection{Materials}

The reagents used in this study were from Merck (Kenilworth, NJ, United States), Acros (Geel, Belgium), and BioRad (Hercules, CA, United Stated). Culture media components were from Difco (Franklin Lakes, NJ, United States) and were used to prepare TB medium (12 g/L tryptone, $24 \mathrm{~g} / \mathrm{L}$ yeast extract, $5 \mathrm{~g} / \mathrm{L}$ glycerol, $23 \mathrm{~g} / \mathrm{L} 170 \mathrm{~g} / \mathrm{L} \mathrm{KH}_{2} \mathrm{PO}_{4}$, and $\left.125 \mathrm{~g} / \mathrm{L} \mathrm{K}_{2} \mathrm{HPO}_{4}\right)$. The crystallisation screens were purchased from Hampton Research (Aliso Viejo, CA, United States) and Molecular Dimensions (Sheffield, UK).

\subsection{Protein Expression and Purification}

E. coli NEB10 $\beta$ cells were transformed with pBAD-based plasmid containing the codon-optimised sequence coding for wild type Pl-LAAO including a His-tag for affinity chromatography located at the $\mathrm{N}$-terminus of the $\mathrm{Pl}$-LAAO sequence. A single colony was used to inoculate $10 \mathrm{~mL} \mathrm{LB}$ preculture which was used, after overnight growth, to inoculate $(1 \% v / v) 1 \mathrm{~L} \mathrm{~TB}_{\mathrm{amp}}$. When the culture reached an $\mathrm{OD}_{600}$ of 0.6 , L-arabinose was added to a final concentration of $0.02 \%(w / v)$ to trigger protein expression. The flasks were moved from 37 to $24{ }^{\circ} \mathrm{C}$ and incubated for 2 days. The obtained cells were harvested by centrifugation $(3153 \times g, 30 \mathrm{~min})$ and the obtained cell pellet was resuspended in $50 \mathrm{mM}$ Tris- $\mathrm{HCl} \mathrm{pH} 7.5$ with $100 \mathrm{mM} \mathrm{NaCl}$ (buffer A). Cells were sonicated for 20 min using 70\% amplitude with 10 $\mathrm{s}$ on/ $5 \mathrm{~s}$ off intervals on a Sonic Vibra cell processor, and cell debris was separated from the supernatant via centrifugation $(20,130 \times g, 30 \mathrm{~min})$. The supernatant was collected, filtered, and applied to pre-equilibrated columns loaded with Ni-Sepharose. After a washing step with $3 \mathrm{CV}$ of buffer $\mathrm{A}$ and the removal of not specifically bound proteins with buffer $\mathrm{A}$ containing $10 \mathrm{mM}$ imidazole (3 CV), the enzyme was eluted using buffer B (buffer A + $500 \mathrm{mM}$ imidazole). The collected sample was desalted using Vivaspin concentrators.

\subsection{Determination of the Extinction Coefficient and Stability}

In order to determine the extinction coefficient of $\mathrm{Pl}$-LAAO, the absorbance spectra of the flavin-containing enzyme and of the FAD after protein denaturation and pellet removal 
were measured at 455 and $450 \mathrm{~nm}$, respectively. The known extinction coefficient of FAD at $450 \mathrm{~nm}\left(11.3 \mathrm{mM}^{-1} \mathrm{~cm}^{-1}\right)$ was used to calculate the extinction coefficient of $\mathrm{Pl}$-LAAO.

The stability of $\mathrm{Pl}$-LAAO was tested using the ThermoFAD protocol [21]. A thermocycler was programmed to run a temperature gradient from 25 to $99{ }^{\circ} \mathrm{C}$ on the samples composed of $12.5 \mu \mathrm{L}$ of different buffers and $12.5 \mu \mathrm{L}$ protein diluted to $1 \mathrm{mg} / \mathrm{mL}$. The emission signal was measured using a SYBR filter (ThermoFisher, Waltham, MA, United States), taking advantage of the increase in FAD fluorescence caused by protein denaturation. The first derivative of the measured peaks provides apparent Tm values.

The residual activity of $\mathrm{Pl}$-LAAO was measured after incubation for $10 \mathrm{~min}$ at different temperatures $\left(30,40,50,60,70,80\right.$, and $\left.90^{\circ} \mathrm{C}\right)$ and over time $(56 \mathrm{~h})$ at $37^{\circ} \mathrm{C}$. The activity was measured using L-phenylalanine as the substrate following the protocol below.

\subsection{Mutagenesis}

The rationally designed mutants of $P l$-LAAO were obtained by Quick Change PCR using pairs of primers designed by AAscan (https: / / github.com/dmitryveprintsev / AAScan, (accessed on 27 October 2021)) to introduce the mutations R87M and Y445F. Successful introduction of mutations was confirmed by sequencing.

\subsection{Kinetic Analysis}

LAAO activity was measured at $37^{\circ} \mathrm{C}$ and both the enzyme samples and the reaction mixtures were preincubated at this temperature. Substrates were added to start the reaction. The final reaction mixture contained $8 \mathrm{U}$ of horseradish peroxidase, $0.1 \mathrm{mM}$ amino antipyrine, $1 \mathrm{mM}$ 3,5-dichloro-2-hydroxybenzene, 180-680 $\mu \mathrm{L}$ substrate at varying concentrations and $0.1 \mu \mathrm{M}-0.5 \mathrm{nM}$ Pl-LAAO in buffer A, for a total volume of $1 \mathrm{~mL}$. Reaction rates of mutants were measured in the same setup, using a fixed concentration of $0.02 \mu \mathrm{M}$ enzyme.

\subsection{Crystallisation and Structure Determination}

For crystallisation, wild type $P l$-LAAO samples underwent further purification by size exclusion chromatography on a Superdex 200 10/300 column (Cytiva, Medemblik, The Netherlands) pre-equilibrated with $100 \mathrm{mM}$ Tris- $\mathrm{HCl}$ pH 7.5 containing $150 \mathrm{mM} \mathrm{NaCl}$ on an ÄKTA purifier (Cytiva). The eluted peak was pooled and concentrated to $24 \mathrm{mg} / \mathrm{mL}$ for crystallisation experiments. Multiple hits were found, with crystals growing in conditions from several commercially available screens. The most easily reproducible crystals were obtained by sitting drop method in 20\% (w/v) PEG 3350 and $0.1 \mathrm{M}$ Tris- $\mathrm{HCl}$ pH 7.0, but also in HEPES pH 7.0 and Tris pH 8.0. The crystals were small cubes of a bright yellow colour, which were cryoprotected by adding $20 \%(v / v)$ glycerol and frozen in liquid nitrogen for synchrotron data collection at Diamond Light Source (Didcot, UK). The collected datasets were merged [22] and scaled [23]. The structure of PDB 7C4K [16] was used as a model for molecular replacement [24]. The obtained model underwent REFMAC5 refinement and manual fitting with COOT [25] yielding the final structure deposited at the PDB with code 7OG2. Images of the Pl-LAAO structure and other models were made using Chimera [26]. The number of salt bridges present in the structure was calculated using the ESBRI tool available on the LSCF Bioinformatics Unit portal [27]. The Pl-LAAO structure was inspected in PyMOL [28] using the CAVER [29] plugin. This allowed analysis of the tunnel leading from the solvent accessible surface to the active site.

\section{Conclusions}

Due to the high expression level, ease of purification, high thermostability, and activity on many different L-amino acids, $\mathrm{Pl}$-LAAO is an attractive enzyme for biocatalysis and biosensing. It can be used for the synthesis of enantiopure amino acids or related compounds. The high catalytic efficiency on a subset of L-amino acids allows it to be used for detection of amino acids. The determined crystal structure can be exploited for tailor-made variants for activity on specific amino acids. 
Supplementary Materials: The following are available online at https:/ /www.mdpi.com/article/ 10.3390/catal11111309/s1, Figure S1: SDS-PAGE of the LAAO purification process. Figure S2: UV-Vis absorbance spectrum of purified LAAO. Figure S3: Size exclusion chromatogram of LAAO sample before crystallisation experiments. The sample was collected in small fractions and pooled together, avoiding the shoulder on the right side of the peak. Figure S4: (A) pH-dependence of Tm of Pl-LAAO in $50 \mathrm{mM}$ TRIS/Cl at different $\mathrm{pH}$ values, (B) salt concentration dependence of Tm of Pl-LAAO in $50 \mathrm{mM}$ TRIS/Cl, pH 6.5, with different $\mathrm{NaCl}$ concentrations, (C) observed PlLAAO activity after $10 \mathrm{~min}$ incubation at different temperature, (D) observed Pl-LAAO activity upon incubation at $37^{\circ} \mathrm{C}$ over time. For the last two figures, data are shown for samples in low salt buffer (blue line) and in high salt buffer (orange line) as comparison. Figure S5: Steady state kinetics for 18 of the 20 proteinogenic amino acids. All measurements were performed in duplicate. No activity was observed for proline and cysteine. Figure S6: Top, sequence alignment between Pl-LAAO and the homologue from ancestral sequence reconstruction, the structure of which is deposited under the PDB 7C4K. Non-conserved sites are highlighted by black bars. Bottom, the structure of 7C4K with non-conserved sites in dark blue. All of these are limited to surface-exposed portions of the protein, while the active site and the cofactor binding residues are fully retained. For sake of clarity, the portions with highest divergence are reported in rectangles of the same colour in both sequence alignment and structure representation. Figure S7: Focus on the electron density (in blue chicken wire) of FAD (in yellow carbons) and glycerol (in green carbons) present only in molecule B of the asymmetric unit. Figure S8: (a) Structure of LAAO with the tunnel (magenta) leading from the FAD cofactor to the solvent-accessible surface. (b) Side view of the tunnel, the entrance of which is enclosed in the black circle. A set of side chains, the motion of which is necessary to allow access to the active site, is highlighted. (c) A $180^{\circ}$ rotated view of the same side view. Table S1: Crystallographic data collection and refinement statistics. The values for the high-resolution shell are shown in parenthesis.

Author Contributions: Conceptualisation, H.L.v.B. and M.W.F.; methodology, S.S., J.D.-M.M. and H.J.R.; writing-original draft preparation, S.S.; writing-review and editing, S.S., H.L.v.B. and M.W.F.; and funding acquisition, M.W.F. All authors have read and agreed to the published version of the manuscript.

Funding: This research was funded by NWO through a VICI grant to M.W.F.

Data Availability Statement: Data are contained within the article.

Conflicts of Interest: The authors declare no conflict of interest.

\section{References}

1. Kasai, K.; Ishikawa, T.; Nakamura, T.; Miura, T. Antibacterial properties of L-amino acid oxidase: Mechanisms of action and perspectives for therapeutic applications. Appl. Microbiol. Biotechnol. 2015, 99, 7847-7857. [CrossRef] [PubMed]

2. Guo, C.; Liu, S.; Yao, Y.; Zhang, Q.; Sun, M.Z. Past decade study of snake venom L-amino acid oxidase. Toxicon 2012, 60, 302-311. [CrossRef] [PubMed]

3. Izidoro, L.F.; Sobrinho, J.C.; Mendes, M.M.; Costa, T.R.; Grabner, A.N.; Rodrigues, V.D.M.; Da Silva, S.L.; Zanchi, F.B.; Zuliani, J.P.; Fernandes, C.F.C.; et al. Snake venom L-amino acid oxidases: Trends in pharmacology and biochemistry. Biomed. Res. Int. 2014, 2014, 196754. [CrossRef]

4. Pawelek, P.D.; Cheah, J.; Coulombe, R.; Macheroux, P.; Ghisla, S.; Vrielink, A. The structure of L-amino acid oxidase reveals the substrate trajectory into an enantiomerically conserved active site. EMBO J. 2000, 19, 4204-4215. [CrossRef] [PubMed]

5. Chen, W.M.; Sheu, F.S.; Sheu, S.Y. Novel L-amino acid oxidase with algicidal activity against toxic cyanobacterium Microcystis aeruginosa synthesized by a bacterium Aquimarina sp. Enzyme Microb. Technol. 2011, 49, 372-379. [CrossRef] [PubMed]

6. Samel, M.; Tõnismägi, K.; Rönnholm, G.; Vija, H.; Siigur, J.; Kalkkinen, N.; Siigur, E. L-Amino acid oxidase from Naja naja oxiana venom. Comp. Biochem. Physiol. Part B 2008, 149, 572-580. [CrossRef] [PubMed]

7. Zuliani, J.P.; Kayano, A.M.; Zaqueo, K.D.; Neto, A.C.; Sampaio, S.V.; Soares, A.M.; Stabeli, R.G. Snake venom L-amino acid oxidases: Some consideration about their functional characterization. Protein Pept. Lett. 2009, 16, 908-912. [CrossRef]

8. Li, Z.Y.; Yu, T.F.; Lian, E.C. Purification and characterization of L-amino acid oxidase from king cobra (Ophiophagus hannah) venom and its effects on human platelet aggregation. Toxicon 1994, 32, 1349-1358. [CrossRef]

9. Lee, M.L.; Tan, N.H.; Fung, S.Y.; Sekaran, S.D. Antibacterial action of a heat-stable form of L-amino acid oxidase isolated from king cobra (Ophiophagus hannah) venom. Comp. Biochem. Physiol. Toxicol. Pharmacol. 2010, 153, 237-242. [CrossRef]

10. Lata, S.; Pundir, C.S. L-amino acid biosensor based on L-amino acid oxidase immobilized onto NiH-CNFe/c-MWCNT/PPy/GC electrode. Int. J. Biol. Macromol. 2013, 54, 250-257. [CrossRef]

11. Kasai, K.; Nakano, M.; Ohishi, M.; Nakamura, T.; Miura, T. Antimicrobial properties of L-amino acid oxidase: Biochemical features and biomedical applications. Appl. Microbiol. Biotechnol. 2021, 105, 4819-4832. [CrossRef] [PubMed] 
12. Huh, J.W.; Yokoigawa, K.; Esaki, N.; Soda, K. Total conversion of racemic pipecolic acid into the L-enantiomer by a combination of enantiospecific oxidation with D-amino acid oxidase and reduction with sodium borohydride. Biosci. Biotechnol. Biochem. 1992, 56, 2081-2082.

13. Busch, F.; Brummund, J.; Calderini, E.; Schürmann, M.; Kourist, R. Cofactor generation cascade for $\alpha$-ketoglutarate and Fe(II)dependent dioxygenases. ACS Sustain. Chem. Eng. 2020, 8, 8604-8612. [CrossRef]

14. Andreo-Vidal, A.; Sanchez-Amat, A.; Campillo-Brocal, J.C. The Pseudoalteromonas luteoviolacea L-amino acid oxidase with antimicrobial activity is a flavoenzyme. Mar. Drugs 2018, 16, 499. [CrossRef]

15. Nakano, S.; Kozuka, K.; Minamino, Y.; Karasuda, H.; Hasebe, F.; Ito, S. Ancestral L-amino acid oxidases for deracemization and stereoinversion of amino acids. Commun. Chem. 2020, 3, 181. [CrossRef]

16. Nakano, S.; Minamino, Y.; Hasebe, F.; Ito, S. Deracemization and stereoinversion to aromatic D-amino acid derivatives with ancestral L-amino acid oxidase. ACS Catal. 2019, 9, 10152-10158. [CrossRef]

17. Pollegioni, L.; Motta, P.; Molla, G. L-amino acid oxidase as biocatalyst: A dream too far? Appl. Microbiol. Biotechnol. 2013, 97, 9323-9341. [CrossRef]

18. Ponnudurai, G.; Chung, M.C.; Tan, N.H. Purification and properties of the L-amino acid oxidase from Malayan pit viper (Calloselasma rhodostoma) venom. Arch. Biochem. Biophys. 1994, 313, 373-378. [CrossRef] [PubMed]

19. Moustafa, I.M.; Foster, S.; Lyubimov, A.Y.; Vrielink, A. Crystal structure of LAAO from Calloselasma rhodostoma with an Lphenylalanine substrate: Insights into structure and mechanism. J. Mol. Biol. 2006, 364, 991-1002. [CrossRef] [PubMed]

20. Umhau, S.; Pollegioni, L.; Molla, G.; Diederichs, K.; Welte, W.; Pilone, M.S.; Ghilsa, S. The x-ray structure of D-amino acid oxidase at very high resolution identifies the chemical mechanism of flavin-dependent substrate dehydrogenation. Proc. Natl. Acad. Sci. USA 2000, 97, 12463-12468. [CrossRef]

21. Forneris, F.; Orru, R.; Bonivento, D.; Chiarelli, L.R.; Mattevi, A. ThermoFAD, a Thermofluor-adapted flavin ad hoc detection system for protein folding and ligand binding. FEBS J. 2009, 276, 2833-2840. [CrossRef] [PubMed]

22. McCoy, A.J.; Grosse-Kunstleve, R.W.; Adams, P.D.; Winn, M.D.; Storoni, L.C.; Read, R.J. Phaser crystallographic software. J. Appl. Crystallogr. 2007, 40, 658-674. [CrossRef]

23. Kabsch, W. XDS. Acta Crystallogr. D Biol. Crystallogr. 2010, 66 Pt 2, 125-132. [CrossRef] [PubMed]

24. Winn, M.D.; Ballard, C.C.; Cowtan, K.D.; Dodson, E.J.; Emsley, P.; Evans, P.R.; Keegan, R.M.; Krissinel, E.B.; Leslie, A.G.W.; McCoy, A.; et al. Overview of the CCP4 suite and current developments. Acta Crystallogr. D Biol. Crystallogr. 2011, 67, $235-242$. [CrossRef] [PubMed]

25. Emsley, P.; Cowtan, K. Coot: Model-building tools for molecular graphics. Acta Crystallogr. D Biol. Crystallogr. 2004, 60, $2126-2132$. [CrossRef] [PubMed]

26. Pettersen, E.F.; Goddard, T.D.; Huang, C.C.; Couch, G.S.; Greenblatt, D.M.; Meng, E.C.; Ferrin, T.E. UCSF Chimera-A visualization system for exploratory research and analysis. J. Comput Chem. 2004, 25, 1605-1612. [CrossRef]

27. Sarakatsannis, J.N.; Duan, Y. Statistical characterization of salt bridges in proteins. Proteins 2005, 60, 732-739. [CrossRef]

28. The PyMOL Molecular Graphics System, version 2.0; Schrödinger, LLC.: New York, NY, USA, 2015.

29. Chovancová, E.; Pavelka, A.; Benes, P.; Strnad, O.; Brezovsky, J.; Kozlikova, B.; Góra, A.; Sustr, V.; Klvana, M.; Medek, P.; et al. CAVER 3.0: A tool for the analysis of transport pathways in dynamic protein structures. PLoS Comput. Biol. 2012, 8, e1002708. [CrossRef] 